Article

\title{
Evolution of Quasiperiodic Structures in a Non-Ideal Hydrodynamic Description of Phase Transitions
}

\author{
D. N. Voskresensky ${ }^{1,2}$ \\ 1 Joint Institute for Nuclear Research, RU-141980 Dubna, Moscow Region, Russia; d.voskresen@gmail.com \\ 2 National Research Nuclear University (MEPhI), Kashirskoe shosse 31, 115409 Moscow, Russia
}

Received: 28 January 2020; Accepted: 3 March 2020; Published: 7 March 2020

check for

updates

\begin{abstract}
Various phase transitions could have taken place in the early universe, and may occur in the course of heavy-ion collisions and supernova explosions, in proto-neutron stars, in cold compact stars, and in the condensed matter at terrestrial conditions. Most generally, the dynamics of the density and temperature at first- and second-order phase transitions can be described with the help of the equations of non-ideal hydrodynamics. In the given work, some novel solutions are found describing the evolution of quasiperiodic structures that are formed in the course of the phase transitions. Although this consideration is very general, particular examples of quark-hadron and nuclear liquid-gas first-order phase transitions to the uniform $k_{0}=0$ state and of a pion-condensate second-order phase transition to a non-uniform $k_{0} \neq 0$ state in dense baryon matter are considered.
\end{abstract}

Keywords: dynamics of phase transitions; spinodal instability; heavy-ion collisions; neutron stars

\section{Introduction}

Cosmological observations of the two last decades [1] have supplied us with some extraordinary results and puzzles. Particularly important is the fact that the universe undergoes an accelerated expansion and the fact that only $5 \%$ of its mass is contained in baryons, $26 \%$ is in dark matter, and the remaining part is in dark energy. It is commonly believed that at least two cosmic phase transitions occurred in the early universe, the electro-weak and the QCD phase transitions [2,3]. The standard model of particle physics predicts that, after the inflation, the hot expanding universe was filled with deconfined quarks in the state of quark-gluon plasma [4]. The quark-gluon plasma in baryon-poor matter persists down to a temperature of $T \simeq 160 \mathrm{MeV}$. Whether the quark-hadron transition is a first-order phase transition, a second-order transition, or a crossover is still not completely settled. This view on the early universe is supported by simulations done in various cosmological and relativistic heavy-ion collision models $[5,6]$ and by the lattice calculations. The latter calculations support the QCD crossover transition obtained by the HotQCD Collaboration $[7,8]$. Nucleosynthesis is affected by remnant inhomogeneities in the baryon-to-entropy ratio and in isospin [9]. These problems can be considered within the standard model. However, the standard model does not account for the presence of the dark matter with which additional cosmic phase transitions may be associated during the cooling of the expanding universe to its present temperature $T \simeq 2.7 \mathrm{~K}$, cf. [10].

Another piece of important information about strongly interacting matter can be extracted from neutrino and photon radiation of compact stars formed in supernova events $[11,12]$ and from analysis of gravitational waves in gamma ray bursts. A strong phase transition may result in a second neutrino burst occurring during a supernova explosion and a hot neutron star formation with a typical minute time delay. It might be also associated with a larger time delay related to the slow heat transport to the neutron-star surface, if the system is close to the pion-condensate phase transition [12,13]. Recently, new arguments have been expressed showing that indeed two neutrino bursts were measured during the 1987A explosion, and that one delayed respectively the other by $4.7 \mathrm{~h}$, cf. [14]. The second burst 
and a blowing of some amount of matter could then be related to the phase transition of the neutron star to the pion-condensate state. A first-order deconfinement transition can serve as an explosion mechanism for massive blue supergiant stars of $M \sim 50 M_{\odot}$, for which so far no explosion mechanism is known [15]. In old neutron stars, the first-order phase transition, if it occurs, could result in a blowing of matter or in a strong star-quake [12,16]. The detection of merging compact stars in the gravitational wave spectra [17] and the detection of massive compact stars [18-21] provide constraints on the equation of state of strongly interacting dense matter and strong phase transitions in it. The dominant postmerger gravitational-wave frequency $f_{\text {peak }}$ may exhibit a significant deviation from an empirical relation between $f_{\text {peak }}$ and the tidal deformability, if a strong first-order phase transition leads to the formation of a gravitationally stable extended quark matter core in the postmerger remnant [22]. Thus, one may feasibly identify observable imprints of a first-order hadron-quark phase transition at supranuclear densities on the gravitational-wave emission of neutron star mergers.

An experimental study of the ultrarelativistic heavy-ion collisions helps to simulate at the terrestrial conditions the processes that have occurred in the very early universe, in supernova explosions and in gamma ray bursts. Experimental data and lattice calculations $[7,8]$ indicate that the hadron-quark transition in heavy-ion collisions at RHIC and LHC collision energies is the crossover transition, cf. [23-25]. For lower collision energies relevant for NICA and FAIR facilities, one expects to find signatures of the strong first-order quark-hadron phase transition [26]. There are experimental evidences that, in the very low-energetic heavy-ion collisions of approximately isospin-symmetrical nuclei, there is a first-order nuclear liquid-gas phase transition (for temperatures $T \lesssim 20 \mathrm{MeV}$ and baryon densities $n \lesssim 0.7 n_{0}$, where $n_{0}$ is the nuclear saturation density) [27-29].

In a many-component system, a mechanical instability is accompanied by a chemical instability, see $[30,31]$. The inclusion of the Coulomb interaction, see $[32,33]$, leads to a possibility of the pasta phase in the neutron star crusts for densities $0.3 n_{0} \lesssim n \lesssim 0.7 n_{0}$. For higher densities in dense neutron star interiors, there may be phase transitions to the pion [12,34], kaon [35,36], and charged rho [37] condensate states and to the quark matter [35,38-41]. The quark-hadron, pion, kaon, and charged rho-meson condensate phase transitions may occur during the iso-entropical falling of the baryon-rich matter in supernova explosions [11], in proto-neutron stars, and in cold compact stars, cf. [12]. In some models, these phase transitions are considered first-order phase transitions leading to mixed phases in dense matter. The formation of the pasta non-uniform phases is one of the possibilities [36,40,41]. We can add here the possibilities of the phase transitions between various superfluid $[42,43]$ and ferromagnetic-superfluid [44] phases in the cold neutron stars and in the color-superconducting hybrid compact stars [45], as well as numerous possibilities of the phase transitions in the condensed matter physics at terrestrial conditions, such as liquid-gas, liquid-glass, and glass-metal transitions.

The liquid-gas phase transition, the transition to the superfluid state in quantum liquids, and many other transitions occur in a uniform state characterized by the wave number $k_{0}=0$. Other phase transitions, such as the transitions in solids and liquid crystals, are transitions to inhomogeneous states characterized by the non-zero wave-vectors, $\vec{k}_{0, i} \neq 0$, cf. $[46,47]$. In glasses, the order, characterized by $k_{0} \neq 0$, appears at rather short distances but disappears at long distances [47]. The phase transition to the pion-condensate state [12,34] possible in the interiors of neutron stars may occur due to a strong $p$-wave pion-baryon attraction, which increases with the increase of the baryon density. Thereby, the pion-condensation occurs in a non-uniform state, $k_{0} \neq 0$. Chiral condensate, which is constant in a vacuum, may also become spatially modulated at high densities, where, in the traditional picture of the QCD phase diagram, a first-order chiral phase transition occurs. Examples of inhomogeneous phases are the chiral density wave, the Skyrme crystal, and crystalline color superconductors, cf. [48]. Perhaps the antikaon condensation in dense baryon matter also occurs in the non-uniform state, $k_{0} \neq 0$, cf. [49].

Some of the mentioned phase transitions, such as the transition of the normal matter to superfluid in metals and in ${ }^{4} \mathrm{He}$, are transitions of the second order [50]. Other phase transitions mentioned above, such as the liquid-gas phase transition, are transitions of the first order. The search for the critical 
endpoint separating the crossover and first-order quark-hadron transitions is one of the benchmarks for future experiments at NICA and FAIR.

Finite size structures are important in the context of the general relativistic evolution of density perturbations in the early universe [10]. Even the simple case of dust baryon matter gives the right order of magnitude for globular star clusters with the corresponding Jeans mass and wavelength. The presence of a substantial amount of homogeneous scalar field energy density at low redshifts inhibits the growth of perturbations in the baryonic fluid [51]. For example, dark matter may result from the transition of a non-minimally coupled scalar field from radiation to collision-less matter. Dynamical instabilities of the field fluctuations, which are typical for oscillatory scalar field regimes, can be amplified and transmitted by the coupling to dark matter perturbations, cf. [52]. The presence of the dark matter may also trigger strong electro-weak phase transition in the early universe [53].

In the early universe, at the processes of the formation of compact stars in supernova explosions, collisions of compact binary stars, and in heavy-ion collisions, one deals with a rapid thermalization of a strongly interacting quark-gluon matter and then the hadronic matter. These processes can be described within non-ideal hydrodynamics, where viscosity and thermal conductivity effects are of crucial importance. The dynamics of the phase transitions can also be considered within non-ideal hydrodynamics, cf. [47,54-56].

Below, some novel solutions will be found describing the evolution of periodic structures at second-order phase transitions to the non-uniform state with the wave number $k_{0} \neq 0$. Quasiperiodic time-dependent structures appear in the course of the spinodal instabilities at the first-order phase transitions to the uniform state with $k_{0}=0$ and in the dynamics of the second-order phase transitions occurring in the uniform state. Although consideration is very general, quark-hadron and nuclear liquid-gas first-order phase transitions and the pion condensation second-order transition will be considered as examples.

The presentation is organized as follows. In Section 2, the main features of the van der Waals-like equation of state are reviewed. In Section 3, a hydrodynamical description of the first- and second-order phase transitions to the uniform, $k_{0}=0$ state and for the second-order phase transition to the nonuniform, $k_{0} \neq 0$ state is formulated assuming a small overcriticality. The dynamics of seeds at the first-order phase transition from a metastable to the stable state is considered in Section 4. The dynamics of fluctuations in the unstable region is studied in Section 5. Some novel solutions describing the time evolution of quasiperiodic and periodic structures are found. Section 6 contains concluding remarks.

\section{Van Der Waals-Like Equation of State for a Description of First-Order Phase Transitions}

The dynamical trajectories of the expanding baryon-rich matter in the heavy-ion collisions and of the falling matter in supernova explosions before phase transition can be characterized by an approximately constant entropy, while the volume $V$ and the temperature $T$ are time-dependent. A description of the first-order phase transition is more involved. In the simplest case of one-component matter, e.g., of the baryon matter, the pressure-baryon number density isotherms $\left.P(n)\right|_{T}$ describing the liquid-like (with a higher density) or gas-like (with a smaller density) states demonstrate a monotonous behavior for the values of temperature $T$ above the critical temperature of the first-order phase transition of the liquid-gas type. However, for $T$ values below the critical temperature, $\left.P(n)\right|_{T}$ isotherms acquire a convex-concave form [47], see Figure 1. The horizontal dashed line connecting points $\mathrm{A}$ and $\mathrm{D}$ shows the Maxwell construction (MC) describing thermal equilibrium of phases. At equilibrium, the baryon chemical potentials are $\mu_{A}=\mu_{D}$. The interval $\mathrm{AB}$ corresponds to a metastable supercooled vapor (SV) and the interval CD relates to a metastable overheated liquid (OL). The interval $\mathrm{BC}$ shows an unstable spinodal region. 


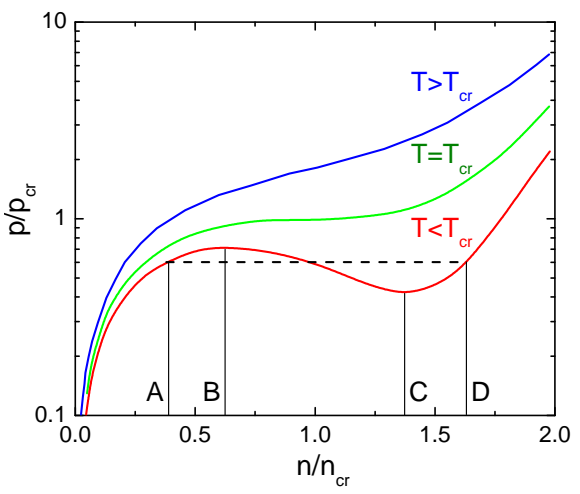

Figure 1. Schematic pressure isotherms as functions of the number density $n$ at a liquid-gas-like phase transition. $P_{c r}, n_{c r}$, and $T_{c r}$ are the pressure, density, and temperature at the critical point.

Adiabatic trajectories $\tilde{s}_{c r}$ and $\tilde{s}_{m}$, where $\tilde{s} \equiv s / n \simeq$ const, and $s$ is the entropy density, are shown in Figure 2 on the plot of $T / T_{c r}=f\left(n / n_{c r}\right)$ by the short dashed lines. The upper convex curve, MC, the bold solid line, demonstrates the boundary of the MC, the bold dashed line, ITS, shows the boundary of the isothermal spinodal region, and the bold dash-dotted curve, AS, indicates the boundary of the adiabatic spinodal region. At the ITS line, $u_{T}^{2}=(\partial P / \partial \rho)_{T}=0$; at the AS line, $u_{\tilde{s}}^{2}=(\partial P / \partial \rho)_{\tilde{s}}=0$, where $u_{T}$ and $u_{\tilde{s}}$ have the meaning of the isothermal and adiabatic sound velocities, respectively, $\rho=m^{*} n$, and $m^{*}$ is the baryon quasiparticle mass. The supercooled vapor (SV) and the overheated liquid (OL) regions are situated between the MC and the ITS curves, on the left and on the right, respectively. For $\tilde{s}_{c r}>\tilde{s}>\tilde{s}_{\mathrm{MC} 2}$, where $\tilde{s}_{c r}$ is the value of the specific entropy $\tilde{s}$ at the critical point and the line with $\tilde{s}_{\mathrm{MC} 2}$ in the example shown in Figure 2 passes through the point $n / n_{c r}=3$ at $T=0$, the system traverses the OL state (the region OL in Figure 2), the ITS region (below the ITS line), and the AS region (below the AS line). For $\tilde{s}>\tilde{s}_{c r}$, the system trajectory passes through the SV state (the region $\mathrm{SV}$ in Figure 2) and the ITS region.

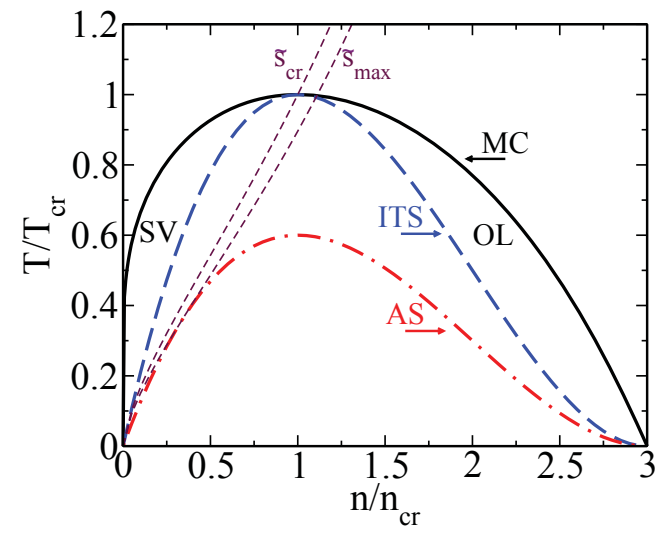

Figure 2. The phase diagram of the van der Waals equation of state on the $T(n)$-plane. The bold solid, dashed, and dash-dotted curves show the boundaries of the MC, the spinodal region at $T=$ const, and $\tilde{s}=$ const, respectively. The short dashed lines show two adiabatic trajectories of the system evolution: the curve labeled $\widetilde{s}_{c r}$ passes through the critical point; $\widetilde{s}_{\text {max }}$ passes through the maximum pressure point $P\left(n_{P, \max }\right)$ on the $P(n)$ plane, cf. [56].

Note that, in reality, for the quark-hadron first-order phase transition, the phase diagram looks slightly different, since then $T_{c r}$ increases with a decrease of the baryon density $[57,58]$. However, this peculiarity does not change a general analysis given here.

When the adiabatic trajectory $\tilde{s}=$ const enters the region of the first-order phase transition (the region below the solid curve in Figure 2), the approximation of the constant entropy fails, and a further description of the dynamics of the system requires a solution of non-ideal hydrodynamical 
equations [54-56]. Similarly, the description of the dynamics of the second-order phase transition requires a solution of non-ideal hydrodynamical equations in the case where the density and the temperature (or entropy) can be considered appropriate order parameters.

\section{Hydrodynamical Description of First- and Second-Order Phase Transitions at Small Overcriticality}

Assume that the dynamics of a second-order phase transition and of a first-order phase transition can be described by the variables $n$ and $s$ (or T), cf. [54-56]. Moreover, assume that the system is rather close to the critical point of the phase transition. Since all the processes in the vicinity of the critical point are slowed down, the velocity of a seed of a new phase prepared in the old phase, $\vec{u}$, is much less than the mean thermal velocity, and thereby we may use equations of non-relativistic non-ideal hydrodynamics: the Navier-Stokes equation, the continuity equation, and the equation for the heat transport, even if we deal with violent heavy-ion collisions:

$$
\begin{aligned}
m^{*} n\left[\partial_{t} u_{i}+(\vec{u} \nabla) u_{i}\right] & =-\nabla_{i} P+\nabla_{k}\left[\eta\left(\nabla_{k} u_{i}+\nabla_{i} u_{k}-\frac{2}{v} \delta_{i k} \operatorname{div} \vec{u}\right)+\zeta \delta_{i k} \operatorname{div} \vec{u}\right] \\
\partial_{t} n+\operatorname{div}(n \vec{u}) & =0 \\
T\left[\frac{\partial s}{\partial t}+\operatorname{div}(s \vec{u})\right] & =\operatorname{div}(\kappa \nabla T)+\eta\left(\nabla_{k} u_{i}+\nabla_{i} u_{k}-\frac{2}{v} \delta_{i k} \operatorname{div} \vec{u}\right)^{2}+\zeta(\operatorname{div} \vec{u})^{2} .
\end{aligned}
$$

Here, as above, $n$ is the number density of the conserving charge, to be specific, the baryon density, $m^{*}$ is the baryon quasiparticle mass, and $P$ is the pressure. The quantities $\eta$ and $\zeta$ are the shear and bulk viscosities, $v=3,2,1$ shows the geometry of the seed under consideration (droplets, rods, and slabs), and $\kappa$ is the thermal conductivity. The treatment of the evolution of seeds within relativistic hydrodynamics is more involved, but this is beyond our scope, cf. [55,59].

All thermodynamical quantities can be expanded near a reference point $\left(n_{\mathrm{r}}, T_{\mathrm{r}}\right)$, which we assume to be close to the critical point but still outside the fluctuation region, which we assume to be narrow. This circumstance is important for the determination of the specific heat density $c_{V, r}$ and, m.b., transport coefficients, which may diverge in the critical point, whereas other quantities are smooth functions of $n, T$, and, by calculating them, one can have that $n_{\mathrm{r}}=n_{c r}, T_{\mathrm{r}}=T_{c r}$.

The Landau free energy, $\delta F_{\mathrm{L}}$, counted from the value at $n_{\mathrm{r}} \simeq n_{c r}, T_{\mathrm{r}} \simeq T_{c r}$ in the variables $\delta n=n-n_{c r}, \delta T=T-T_{c r}$, and $\delta\left(\delta F_{L}\right) / \delta(\delta n)=P-P_{f}+P_{\mathrm{MC}}$, can be presented as [54-56]

$$
\delta F_{\mathrm{L}}=\int \frac{d^{3} x}{n_{c r}}\left\{\frac{c m^{*}[\nabla(\delta n)]^{2}}{2}+\frac{\lambda m^{*}(\delta n)^{4}}{4}-\frac{\lambda v^{2} m^{*}(\delta n)^{2}}{2}-\epsilon \delta n\right\}+\delta F_{\mathrm{L}}\left(k_{0}\right),
$$

where $\epsilon=P_{f}-P_{\mathrm{MC}} \simeq n_{\mathrm{cr}}\left(\mu_{i}-\mu_{f}\right)$ is expressed through the (final) value of the pressure after the first-order phase transition has occurred, and the pressures at the $\mathrm{MC}, \mu_{i}$ and $\mu_{f}$, are the chemical potentials of the initial and final configurations (at fixed $\mathrm{P}$ and $\mathrm{T}$ ). The quantity $\epsilon \neq 0$ if one deals with a first-order phase transition, and $\epsilon=0$ if a transition is of the second order. The maximum of the quantity $\epsilon$ is $\epsilon_{m}=4 \lambda v^{3} /(3 \sqrt{3})$. For the description of phase transitions to the uniform state, $k=0$, one may retain only the term $\propto c[\nabla(\delta n)]^{2}$ in the expansion of the free energy in the density gradients using $c>0$. For the description of phase transitions to the non-uniform state, $k_{0} \neq 0$, one should perform expansion retaining terms at least up to $\propto d[\Delta(\delta n)]^{2}$ assuming $c<0$ and $d>0$. Therefore, the last term in Equation (4) appears only if $k=k_{0} \neq 0$ [47], as is the case for the phase transition to the solid state, liquid crystal state, or pion-condensate state in dense nuclear matter. Thus, for $k_{0} \neq 0$ and $c<0, d>0$, we have

$$
\delta F_{\mathrm{L}}\left(k_{0}\right)=\int \frac{d^{3} x}{n_{c r}}\left\{\frac{d m^{*}}{2}(\Delta \delta n)^{2}-\left(\frac{c m^{*} k_{0}^{2}}{2}+\frac{d m^{*} k_{0}^{4}}{2}\right)(\delta n)^{2}\right\}
$$


where $k_{0}^{2}=-\frac{c}{2 d}>0$ follows from the minimization of $\delta F_{\mathrm{L}}\left(k_{0}\right)$. In the case of the phase transition to the uniform state, one should have $k_{0}=0, d=0$ (then $\delta F_{\mathrm{L}}\left(k_{0}\right)=0$ ), and $c>0$. Thus, the first term $\propto c$ in Equation (4) is associated with the positive surface tension, $\delta F_{\mathrm{L}}^{\text {surf }}=\sigma S$, where $S$ is the surface of the seed.

The Landau free energy density and pressure as functions of the order parameter $\delta \rho / v$ for the equation of state determined by Equation (4) are shown in Figure 3. For $\epsilon=0$, two minima of the Landau free energy coincide and correspond to the MC on the curve $\delta P(1 / \rho)$ (shown by horizontal lines in the plot $\delta P(\delta \rho)$ in the right panel). If, in the initial state, $(\delta \rho)_{i}=\rho_{i}-\rho_{c r}=0$, we deal with the spontaneous symmetry breaking and the second-order phase transition. For $(\delta \rho)_{i}=\rho_{i}-\rho_{c r} \neq 0$, $\epsilon>0$, or $\epsilon<0$, we deal either with the first-order phase transition from the metastable to the stable state, if $\rho_{i}$ corresponds to the metastable state, or with the second-order phase transition either to the metastable state or to the stable state. For $\epsilon>0$ (solid lines), the liquid state is stable and the gas state is metastable (SV); for $\epsilon<0$ (dash-dotted lines), the liquid state is metastable (OL), whereas the gas state is stable. The dynamics of the transition starting from a point within the spinodal region for $\epsilon \neq 0$ (but small) is described similarly to that for the second-order phase transition for $\epsilon=0$.
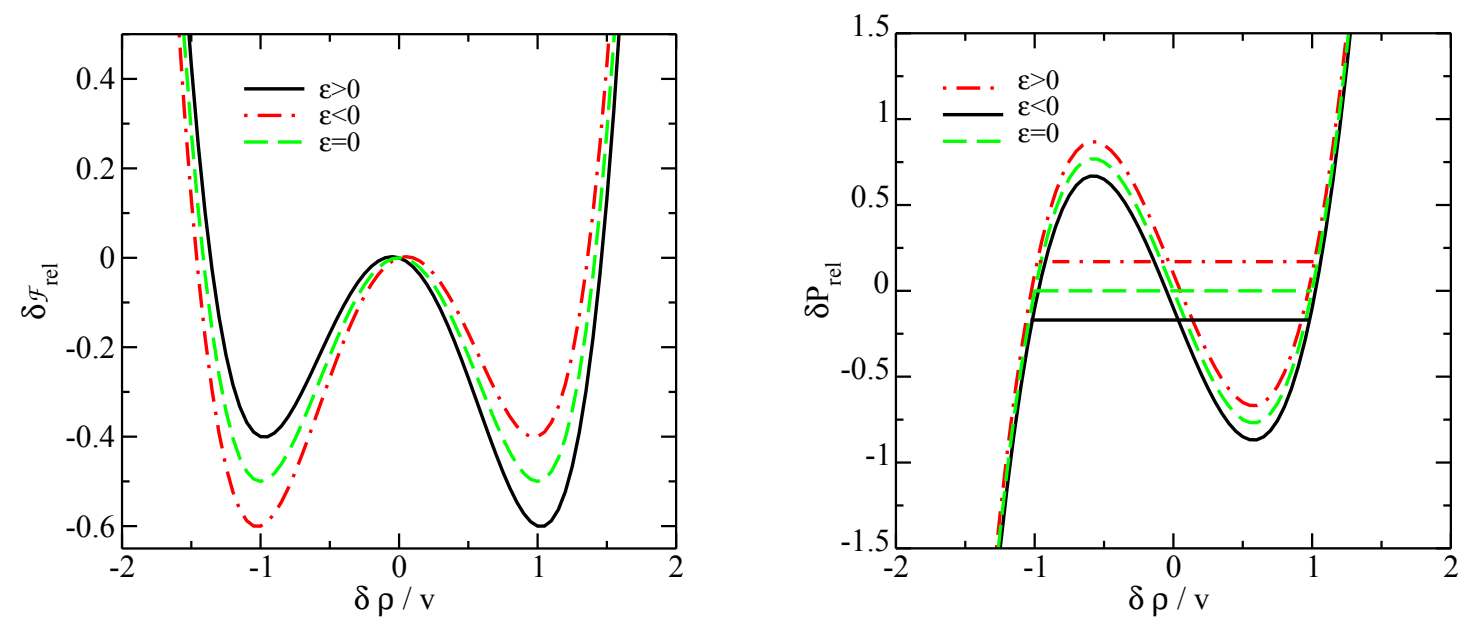

Figure 3. The Landau free energy density $\delta \mathcal{F}_{\text {rel }}=\delta \mathcal{F}_{\mathrm{L}} / \mathcal{F}_{\mathrm{L}}\left(T_{c r}, \rho_{c r}\right)$ and the value $\delta P_{\text {rel }}=$ $\left.\rho_{c r} \frac{\delta\left[F_{\mathrm{L}}(T, \delta \rho)\right]}{\delta(\delta \rho)}\right|_{T} / P\left(T_{c r}, \rho_{c r}\right)$, as functions of the order parameter $\delta \rho=m^{*} \delta n$ for the EoS determined by Equation (4), at $T<T_{c r}$. The dashed horizontal line $(\epsilon=0)$ in the right panel shows MC, cf. [55].

For the purely van der Waals equation of state (in this case, $k_{0}=0$ ), one obtains [55]:

$$
v^{2}(T)=-4 \frac{\delta T n_{c r}^{2} m^{* 2}}{T_{c r}}, \quad \sigma=\sigma_{0} \frac{|\delta T|^{3 / 2}}{T_{c r}^{3 / 2}}, \quad \sigma_{0}^{2}=32 m^{*} n_{c r}^{2} T_{c r} c
$$

Applying operator div to Equation (1) and replacing $\operatorname{div} \vec{u}$ from Equation (2) for small $\delta \rho$ and $u$, keeping only linear terms in $u$, which is legitimate, since near the critical point processes develop slowly $\left(v^{2} \propto-\delta T\right)$, we rewrite Equation (1) as

$$
\begin{gathered}
-\frac{\partial^{2} \delta n}{\partial t^{2}}=\Delta\left[c \Delta \delta n+\lambda v^{2} \delta n-\lambda m^{* 2}(\delta n)^{3}+\epsilon / m^{*}-\left(m^{*} n_{c r}\right)^{-1}\left(\widetilde{v} \eta_{c r}+\zeta_{c r}\right) \frac{\partial \delta n}{\partial t}\right] \\
-\Delta\left[d \Delta^{2} \delta n+\left(c k_{0}^{2}+d k_{0}^{4}\right) \delta n\right]
\end{gathered}
$$

$\widetilde{v}=2(v-1) / v$, cf. $[12,47,55]$. The second line in Equation (7) yields a non-zero term only for the description of the condensation to the inhomogeneous state. 
Consider $T<T_{c r}$. In the dimensionless variables $m^{*} \delta n=v \psi, \tau=t / t_{0}, \xi_{i}=x_{i} / l, i=1, \cdots, v$, $v=3$ for seeds of spherical geometry, Equation (7) is presented as

$$
\begin{aligned}
& -\beta \frac{\partial^{2} \psi}{\partial \tau^{2}}=\Delta_{\xi}\left(\Delta_{\xi} \psi+2 \psi\left(1-\psi^{2}\right)+\widetilde{\epsilon}-\frac{\partial \psi}{\partial \tau}-\frac{\lambda v^{2} d}{2 c^{2}} \Delta_{\tilde{\zeta}}^{2} \psi+\frac{2\left(c k_{0}^{2}+d k_{0}^{4}\right)}{\lambda v^{2}} \psi\right), \\
& l=\left(\frac{2 c}{\lambda v^{2}}\right)^{1 / 2}, t_{0}=\frac{2 \tilde{\eta}_{\mathrm{r}}}{\lambda v^{2}}, \widetilde{\epsilon}=\frac{2 \epsilon}{\lambda v^{3}}, \beta=\frac{c}{\tilde{\eta}_{\mathrm{r}}^{2}}, \quad \tilde{\eta}_{\mathrm{r}}=\frac{\left(\tilde{\nu} \eta_{\mathrm{r}}+\zeta_{\mathrm{r}}\right)}{m^{*} n_{c r}} .
\end{aligned}
$$

It is important to notice that, even for $k_{0}=0$, Equation (8) differs in form from the standard Ginzburg-Landau equation broadly exploited in the condensed matter physics, since Equation (8) is of the second order in time derivatives, whereas the standard Ginzburg-Landau equation is of the first order in time derivatives. The difference disappears if one sets the bracketed-term in the r.h.s. of Equation (8) to zero. Such a procedure is, however, not legitimate at least for a description of the order parameter on an initial time-stage, since two initial conditions, such as $\delta n(t=0, \vec{r})=0$ and $\left.\partial_{t} \delta n(t, \vec{r})\right|_{t=0} \simeq 0$, should be fulfilled to describe the evolution of an initially formed fluctuation (seed). Thereby, there exists at least an initial stage of the dynamics of seeds (for $t \lesssim t_{\text {init }}$ ), which is not described by the standard Ginzburg-Landau equation [54,55]. The bracketed term in the r.h.s. of Equation (8) can indeed be set to zero, see below, if one considers an effectively very viscous medium at $\tau \gg 1$. Note also that Equation (8), with the bracketed term in the r.h.s. equal to zero, can be derived from the first-gradient order kinetic equation of Kadanoff-Baym [60].

Equation (8) should be supplemented by Equation (3) for the heat transport, which, owing to Equation (2) after its linearization, reads as

$$
T_{c r}\left[\partial_{t} \delta s-s_{c r}\left(n_{c r}\right)^{-1} \partial_{t} \delta n\right]=\kappa_{\mathrm{r}} \Delta \delta \mathrm{T} .
$$

The variation of the temperature is related to the variation of the entropy density $s[n, T]$ by

$$
\delta T \simeq T_{c r}\left(c_{V, \mathrm{r}}\right)^{-1}\left(\delta s-(\partial s / \partial n)_{T, c r} \delta n\right),
$$

where $c_{V}$ is the density of the heat capacity.

\subsection{Typical Time Scales}

Let us perform some rough dimensional estimates of typical time scales in the problem. The evolution of a seed of one phase in another phase is governed by the slowest mode $(\delta \rho$ or $\delta$, respectively). The time scale for the relaxation of the density following Equation (8) is $t_{0} \propto \tilde{\eta}$. Thus, the non-zero viscosity plays the role of the driving force managing the time evolution of the density mode. Moreover, $t_{0} \propto 1 /\left(T_{c r}-T\right)$. Thereby, the processes are slowed down near the critical point of the phase transition. The time scale for the relaxation of the entropy/temperature mode, following Equation (9), is

$$
t_{T}=R_{\text {seed }}^{2} c_{V, \mathrm{r}} / \kappa_{\mathrm{r}} \propto R_{\text {seed }}^{2}
$$

i.e., the relaxation time of the temperature/entropy is proportional to the surface of the seed. Thus, for $t_{T}\left(R_{\text {seed }}\right)<t_{0}$, i.e for $R_{\text {seed }}<R_{\text {fog }}=\sqrt{\kappa_{\mathrm{r}} t_{0} / c_{V, r}}$, where $R_{\text {fog }}$ is the typical size of the seed at $t \sim t_{0}=t_{T}$, the dynamics of the seeds is controlled by Equation (8) for the density mode. For seeds with sizes $R_{\text {seed }}>R_{\text {fog }}$, the quantity $t_{T} \propto R_{\text {seed }}^{2}$ exceeds $t_{0}$, and the growth of seeds is slowed down. Thereby, the number of seeds with the typical size $R_{\text {seed }} \sim R_{\text {fog }}$ is increased with the passage of time, and a state of fog is formed. For the quark-hadron phase transition in energetic heavy-ion collisions, one [55] estimates $R_{\text {fog }} \sim(0.1-1) \mathrm{fm}$ and, for the nuclear liquid-gas transition at low energies, $R_{\text {fog }} \sim(1-10) \mathrm{fm} \lesssim R\left(t_{\text {f.o. }}\right)$, where $R\left(t_{\text {f.o. }}\right)$ is the size of the fireball at the freeze-out, and $t_{\text {f.o. }}$ is the fireball evolution time until freeze-out. 
There are only two dimensionless parameters in Equation (8): $\widetilde{\epsilon}$ and $\beta$. The parameter $\widetilde{\epsilon}$ is responsible for a difference between the Landau free energies of the metastable and stable states. For $t_{0} \gg t_{T}$ (the isothermal stage), $\widetilde{\epsilon} \simeq$ const and the dependence on this quantity disappears because of $\Delta_{\zeta} \widetilde{\epsilon} \simeq 0$. Then, the dynamics is controlled by the parameter $\beta$, which characterizes the inertia. It is expressed in terms of the surface tension and the viscosity as

$$
\beta=\left(32 T_{c r}\right)^{-1}\left[\widetilde{v} \eta_{\mathrm{r}}+\zeta_{\mathrm{r}}\right]^{-2} \sigma_{0}^{2} m^{*}
$$

The larger viscosity and the smaller surface tension, the effectively more viscous (inertial) is the fluidity of seeds. For $\beta \ll 1$, one deals with the regime of effectively viscous (inertial) fluidity and at $\beta \gg 1$, one deals with the regime of almost perfect fluidity. Estimates [55] show that, for the nuclear liquid-gas phase transition, typically $\beta \sim 0.01$. For the quark-hadron transition, $\beta \sim 0.02-0.2$, even for a very low value of the $\eta / s \simeq 1 /(4 \pi)$ ratio. The latter quantity characterizes the fluidity of the matter at ultra-relativistic heavy-ion collisions [25]. Thus, as we argued, in the case of baryon-rich matter, one effectively deals with a very viscous (inertial) evolution of density fluctuations, in cases of nuclear liquid-gas and quark-hadron phase transitions.

In neutron stars, an overcritical pion-condensate drop reaches a size $R \sim 0.1 \mathrm{~km}$ for $t \sim 10^{-3} \mathrm{~s}$ by the growth of the density mode. Then, it may reach $R \sim(1-10) \mathrm{km}$ for typical time $t_{T}$ varying from $\sim 10 \mathrm{~s}$ for up to several hours (rather than for a typical collapse time $\sim 10^{-3} \mathrm{~s}$ ). A delay appears owing to neutrino heat transport to the surface (an effect of neutrino thermal conductivity), and this delay strongly depends on the value of the pion softening, which is stronger for most massive neutron stars [12]. One should also take into account that the bulk viscosity is significantly increased in the presence of soft modes [61,62], e.g., near the pion condensation critical point [63]. Notice also that the description of the dynamics of the pion-condensate phase transition is specific, since the transition occurs to the inhomogeneous liquid-crystal-like state characterized by $\vec{k} \neq 0$. The seeds of the liquid-crystal-like state prove to be elongated in the process of their growth [47]. A similar effect is observed in liquid crystals.

Thus, the interplay between viscosity, surface tension, and thermal conductivity effects is responsible for the typical time and size scales of fluctuations.

\subsection{Stationary Solutions}

Now let us find stationary solutions of Equation (7). For the condensation in the state $k \neq 0$, we find a solution in the form

$$
m^{*} \delta n=a\left[\sin (k x+\chi)+\frac{c_{1} \widetilde{\omega}^{2}\left(k^{2}\right)}{\widetilde{\omega}^{2}\left(9 k^{2}\right)} \sin (3 k x+\chi)+\ldots\right]+O(\epsilon),
$$

where $\chi$ is a constant phase,

$$
\widetilde{\omega}^{2}\left(k^{2}\right)=-\lambda v^{2}+c k^{2}+d k^{4}-c k_{0}^{2}-d k_{0}^{4} .
$$

For the condensation in the uniform state $k_{0} \neq 0, c<0$, and $d>0$, the gap $\widetilde{\omega}^{2}\left(k^{2}\right)$ has a minimum for $k=k_{0}$. The phase transition arises for $\widetilde{\omega}^{2}\left(k_{0}^{2}\right)<0$. Setting Equation (13) in Equation (7), we find

$$
a^{2}=-\frac{4}{3} \widetilde{\omega}^{2}\left(k_{0}^{2}\right) / \lambda>0, \quad c_{1}=-1 / 3
$$

Minimization of the free energy in $k$ yields $k=k_{0} \cdot \widetilde{\omega}^{2}\left(k_{0}^{2}\right)=-\lambda v^{2}$, and $\widetilde{\omega}^{2}\left(k_{0}^{2}\right)>0$ for $T>T_{c r}$. $\widetilde{\omega}^{2}\left(k_{0}^{2}\right)<0$ for $T<T_{c r} \cdot \widetilde{\omega}^{2}\left(9 k_{0}^{2}\right)=-\lambda v^{2}+16 c^{2} / d \gg\left|\widetilde{\omega}^{2}\left(k_{0}^{2}\right)\right|$. Thereby, with appropriate accuracy, we may use $\delta n \simeq a\left[\sin \left(k_{0} x+\chi\right)\right.$, which yields $\delta F_{L}\left(k_{0}\right) \simeq-\lambda v^{4} V /\left(6 m^{*} n\right)+O\left(\epsilon^{2}\right)$, where $\mathrm{V}$ is the volume of the system. Thus, the solution expressed in Equation (13) describes the stationary state at the second-order phase transition. 
For the condensation in the uniform state $k_{0}=0$, we have [55]

$$
\widetilde{\omega}^{2}\left(k^{2}\right)=-\lambda v^{2}+c k^{2}, \quad k^{2}<\lambda v^{2} / c, \quad c>0 .
$$

Two spatially constant stationary solutions minimizing the free energy for $T<T_{c r}$ correspond to $k=0$. They describe metastable and stable states:

$$
\delta n_{\mathrm{st}} \simeq \pm v / m^{*}+\epsilon /\left(2 \lambda v^{2} m^{*}\right)
$$

The free energy corresponding to these solutions is given by

$$
\delta F_{\mathrm{L}}\left(k=0, k_{0}=0\right) \simeq-\frac{\lambda v^{4} V}{4 m^{*} n_{c r}}\left(1 \pm \frac{4 \epsilon}{\lambda v^{3}}\right) .
$$

For $k \neq 0$, solutions in the form of Equation (13) are valid for $\left|\widetilde{\omega}^{2}\left(k^{2}\right)\right| \ll \widetilde{\omega}^{2}\left(9 k^{2}\right)$. For $k_{0}=0$, they yield

$$
\delta F_{\mathrm{L}}\left(k \neq 0, k_{0}=0\right) \simeq-\frac{\lambda v^{4}\left(1-c k^{2} /\left(\lambda v^{2}\right)\right) V}{6 m^{*} n_{c r}} .
$$

Although the minimum of the free energy for $k_{0}=0$ is given by Equation (18), corresponding to solutions expressed in Equation (17) obtained for $k=0$ rather than by solutions expressed in Equation (13) corresponding to the free energy expressed in Equation (19), as we will demonstrate below, solutions expressed in Equation (13) characterized by $k \neq 0$ have a physical meaning.

\section{Dynamics of Seeds at a First-Order Phase Transition from a Metastable State to a Stable State}

The rate of the formation of seeds in fluctuations has been extensively studied in the literature, e.g., $[9,59,64-66]$. Let us assume that an initial seed of the new phase has been formed in a fluctuation and consider its subsequent time evolution. Consider the limit of a high thermal conductivity, when, in Equation (7), the temperature can be made constant. The solution expressed in Equation (7) describing the dynamics of the initial density fluctuation developing from the metastable state to the stable state is then presented in the form [56]

$$
\delta n(t, r) \simeq \frac{v(T)}{m}\left[ \pm \operatorname{th} \frac{r-R_{\text {seed }}(t)}{l}+\frac{\epsilon}{2 \lambda v^{3}(T)}\right]+(\delta n)_{\mathrm{cor}}
$$

where the upper sign corresponds to the evolution of bubbles of the gas, the lower sign solution describes the evolution of droplets of liquid for $v=3$, and the solution is valid for $\left|\epsilon /\left(\lambda v^{3}(T)\right)\right| \ll 1$. Compensating correction $(\delta n)_{\text {cor }}$ is introduced to fulfill the baryon number conservation. Considering spatial coordinate $r$ in the vicinity of a bubble/droplet boundary, we obtain an equation describing the evolution of the seed size [55,56]:

$$
\frac{m^{* 2} \beta t_{0}^{2}}{2 l} \frac{d^{2} R_{\text {seed }}}{d t^{2}}=m^{* 2}\left[\frac{3 \epsilon}{2 \lambda v^{3}(T)}-\frac{2 l}{R_{\text {seed }}}\right]-\frac{m^{* 2} t_{0}}{l} \frac{d R_{\text {seed }}}{d t}
$$

This equation reminds us of Newton's second law for a one-dimensional system, where the quantity $M=\frac{m^{* 2} \beta t_{0}^{2}}{2 l} \propto\left(T_{c r}-T\right)^{-3 / 2}$ has the meaning of the mass, $m^{* 2}\left[\frac{3 \epsilon}{2 \lambda v^{3}(T)}-\frac{2 l}{R_{\text {seed }}}\right]$ is an external force, and $-\frac{m^{* 2} t_{0}}{l} \frac{d R_{\text {seed }}}{d t}$ is the friction force, with a viscous-friction coefficient that is proportional to an effective viscosity and inversely proportional to $\sqrt{T_{c r}-T}$. Following Equation (21), a bubble of an overcritical size $R_{\text {seed }}>R_{c r}=4 l \lambda v^{3}(T) /(3 \epsilon)$ of the stable gas phase, or respectively a droplet of the stable liquid phase, is initially prepared in a fluctuation inside a metastable phase and then grows. In an early stage of the evolution, the size of the overcritical bubble/droplet $R_{\text {seed }}(t)$ (for $R_{\text {seed }}>R_{c r}$ ) grows with acceleration. Thus, it reaches a steady growth regime with a constant velocity 
$u_{\mathrm{as}}=\frac{3 \epsilon l}{\lambda v^{3}(T) t_{0}} \propto\left|\left(T_{c r}-T\right) / T_{c r}\right|^{1 / 2}$. In the interior of the seed $\delta n \simeq \mp v(T) / m^{*}$. The correction $(\delta n)_{\text {cor }} \simeq v(T) R_{\text {seed }}^{3}(t) /\left(m^{*} R^{3}\right)$ is very small for $R_{\text {seed }}(t) \ll R$, where $R$ is the radius of the whole system. In cases of the quark-hadron and nuclear liquid-gas phase transitions in heavy-ion collisions, $R(t)$ is the radius of the expanding fireball. Usage of the isothermal approximation in Equation (20) needs the fulfillment of inequality $t_{\rho} \sim \frac{R_{\text {seed }}\left(t_{\text {f.o. }}\right)}{u_{\text {as }}} \gg t_{T}$. For $R_{\text {seed }} \sim R_{c r}$ and for $\epsilon \sim \epsilon_{m}$, we obtain $t_{\rho} \sim t_{0}$, and the isothermal approximation is valid for $R_{\text {seed }}<R_{\text {fog }}$. For $\epsilon \ll \epsilon_{m}$, we obtain $t_{\rho} \gg t_{0}$, and the isothermal approximation remains correct for seeds of the size $R_{\text {seed }}<R_{\mathrm{fog}} \epsilon_{m} / \epsilon$.

Substituting Equation (20) in Equation (9) for $T \simeq$ const (which is correct in linear approximation), we obtain

$$
\delta s=\left(\frac{\partial s}{\partial n}\right)_{T}\left\{\frac{v(T)}{m}\left[ \pm \mathrm{th} \frac{r-R_{\text {seed }}(t)}{l}+\frac{\epsilon}{2 \lambda_{c r} v^{3}(T)}\right]+(\delta n)_{\text {cor }}\right\} .
$$

Note that, for the description of the expanding fireball formed in heavy-ion collisions, the approximation of a quasi-adiabatic expansion can be used even in the presence of a weak first-order phase transition (for $\delta s \ll s$ and $\delta n \ll n$ ). The evolution of droplets/bubbles in the metastable region can be considered at a fixed size of the fireball, provided an expansion time lasting until freeze-out $t_{\text {f.o. }} \gg\left(t_{\rho}, t_{T}\right)$.

\section{Dynamics of Fluctuations in Unstable Region}

\subsection{Growth of Fluctuations of Small Amplitude. Linear Regime}

In this section, the " $r$ " reference point can be taken as arbitrary, so we suppress the subscript " $r$. " To find solutions of the linearized hydrodynamical equations, we have, cf. [56],

$$
\delta n=\delta n_{0} \exp [\gamma t+i \vec{k} \vec{r}]-\frac{\epsilon}{m^{*} \lambda v^{2}}, \quad \delta s=\delta s_{0} \exp [\gamma t+i \vec{k} \vec{r}], \quad T=T_{>}+\delta T_{0} \exp [\gamma t+i \vec{k} \vec{r}],
$$

where $T_{>}$is the temperature of the uniform matter. For $|\delta n| \gg\left|\frac{\epsilon}{m^{*} \lambda v^{2}}\right|$, i.e., for $\epsilon \ll \epsilon_{m}$, the description of the fluctuation in the spinodal region at the first-order phase transition and the description of the second-order phase transition are the same. We may set $\epsilon \rightarrow 0$. Thus, from linearized equations of non-ideal hydrodynamics, expressed in Equations (7) and (9), we find the increment $\gamma(k)$, cf. [56],

$$
\gamma^{2}=-k^{2}\left[\widetilde{\omega}^{2}\left(k^{2}\right)+\tilde{\eta} \gamma+\frac{u_{\tilde{s}}^{2}-u_{T}^{2}}{1+\kappa k^{2} /\left(c_{V} \gamma\right)}\right],
$$

where $\tilde{\eta}=\frac{(\tilde{\nu} \eta+\zeta)}{m^{*} \eta}$. This equation differs from that derived in [67] by the presence of an extra surface tension term, and it differs from that in [59], which was based on other assumptions. Equation (24) has three solutions corresponding to the growth of the density and thermal modes. For $k k^{2} /\left(c_{V}|\gamma|\right) \gg 1$, the temperature in the seed can be made constant, and we may deal with only one equation for the density mode expressed in Equation (7), which yields

$$
\gamma^{2}=-k^{2}\left[\widetilde{\omega}^{2}\left(k^{2}\right)+\tilde{\eta} \gamma\right]
$$

from which we find two solutions for the density modes,

$$
\gamma_{1,2}=-\frac{k^{2} \tilde{\eta}}{2} \pm \sqrt{\frac{k^{4} \tilde{\eta}^{2}}{4}-k^{2} \tilde{\omega}^{2}\left(k^{2}\right)}
$$


For $\left.\omega^{2} \tilde{(} k^{2}\right)<0$, which corresponds to the region of the phase transition, the upper-sign solution, $\gamma_{1}>0$, describes the growing mode, and the lower sign solution, $\gamma_{2}<0$, describes the damping mode. For $k^{2} \tilde{\eta}^{2} /\left|\tilde{\omega}^{2}\left(k^{2}\right)\right| \ll 1$, we have

$$
\gamma_{1} \simeq \sqrt{-k^{2} \tilde{\omega}^{2}\left(k^{2}\right)}-\frac{k^{2} \tilde{\eta}}{2}+O\left(k^{3} \tilde{\eta}^{2} /\left|\tilde{\omega}\left(k^{2}\right)\right|\right)
$$

for the growing mode. In the opposite limit $k^{2} \tilde{\eta}^{2} /\left|\tilde{\omega}^{2}\left(k^{2}\right)\right| \gg 1$, we obtain

$$
\gamma_{1} \simeq-\tilde{\omega}^{2}\left(k^{2}\right) / \tilde{\eta}+O\left(\tilde{\omega}^{4}\left(k^{2}\right) /\left(k^{2} \tilde{\eta}^{3}\right)\right) .
$$

Note that, in condensed matter physics, a transition from a liquid to a glass state can be interpreted as a first-order phase transition occurring within a spinodal region at a very high viscosity [47]. Thus, there is an order at a scale of several $\AA$, which transforms in a disorder at larger distances.

For $k_{0}=0, c>0$, for the most rapidly growing mode (for $\gamma_{m}=\max \left\{\gamma_{1}\right\}$ corresponding to $\left.k=k_{m}\right)$, we find

$$
\gamma_{m} \simeq \frac{\lambda v^{2}}{(2 \sqrt{\beta}+1) \tilde{\eta}}, \quad k_{m}^{2} \simeq \frac{\lambda v^{2} \sqrt{\beta}}{(2 \sqrt{\beta}+1) c} .
$$

For $k_{0} \neq 0, c<0, d>0$, the most rapidly growing mode corresponds to $k=k_{0}$; thus, $\tilde{\omega}^{2}\left(k_{0}^{2}\right)<0$ and $\left|\tilde{\omega}^{2}\left(k_{0}^{2}\right)\right|$ as a function of $k^{2}$ are the largest.

\subsection{The Growth of Fluctuations of Arbitrary Amplitude: A Nonlinear Regime}

Now we will find the solution to the non-linear Equation (7). We search the solution in the form

$$
m^{*} \delta n=a f(t)\left[\sin (k x+\chi)+\frac{c_{1} \widetilde{\omega}^{2}\left(k^{2}\right)}{\widetilde{\omega}^{2}\left(9 k^{2}\right)} \sin (3 k x+\chi)+\ldots\right]+O(\epsilon),
$$

as Equation (13) with $a^{2}=-\frac{4 \widetilde{\omega}^{2}}{3 \lambda}>0$, but now with $f(t)$, satisfying

$$
\partial_{t}^{2} f=-k^{2} \widetilde{\omega}^{2}\left(k^{2}\right) f\left(1-f^{2}\right)-k^{2} \tilde{\eta} \partial_{t} f .
$$

For $k^{2} \tilde{\eta}^{2} /\left|\tilde{\omega}^{2}\left(k^{2}\right)\right| \gg 1$, i.e., for $\beta \ll 1$ or $\tilde{\eta} \gg \sqrt{c}$, the term $\partial_{t}^{2} f$ on the 1.h.s. of Equation (30) can be dropped, and the amplitude

$$
f(t)=\frac{f_{0} e^{\gamma t}}{\sqrt{1+f_{0}^{2} e^{2 \gamma t}}}
$$

fulfills the resulting Equation (30). $f_{0} / \sqrt{1+f_{0}^{2}}$ shows the amplitude of the fluctuation at $t=0$, and $f_{0}$ is an arbitrary constant. For $k \sim k_{m}$ at $k_{0}=0$, this solution holds for $k_{m}^{2} \tilde{\eta}^{2} /\left|\tilde{\omega}^{2}\left(k_{m}^{2}\right)\right| \gg 1$. For $k=k_{0} \neq 0$, the criterion of applicability renders as $k_{0}^{2} \tilde{\eta}^{2} /\left|\tilde{\omega}^{2}\left(k_{0}^{2}\right)\right| \gg 1$. In both cases $k_{0}=0$ and $k_{0} \neq 0$, with the density distribution given by Equations (29) and (31), the free energy renders

$$
\delta F_{\mathrm{L}}(t)=-\frac{V \widetilde{\omega}^{4}\left(k^{2}\right)}{6 \lambda m^{*} n} f^{2}(t)\left(2-f^{2}(t)\right) .
$$

For $t \rightarrow \infty$, we have $f(t \rightarrow \infty) \rightarrow 1$, and $\delta F_{\mathrm{L}}$ reaches the minimum. For $k=k_{0}$, this value coincides with Equation (19), which is given by the stationary solution.

In the general case, Equation (31) yields an interpolation between two approximate solutions that are valid for the limit cases $\gamma t \ll 1$ and $\gamma t \gg 1$. Replacing Equation (31) in Equation (30), we obtain then the same solutions expressed in Equation (26) as those in the linear case. 
Let first $k_{0}=0$. For $t \rightarrow \infty$, using Equation (31) at $\gamma=\gamma_{m}=\gamma\left(k_{m}\right)$, we find

$$
\delta F_{\mathrm{L}}(t \rightarrow \infty)=-\frac{\widetilde{\omega}^{4}\left(k_{m}^{2}\right) V}{6 \lambda m^{*} n} .
$$

For the case of a large effective viscosity/inertia, $\beta \ll 1$, we obtain $\delta F_{\mathrm{L}}(t \rightarrow \infty) \simeq-\frac{\lambda v^{4} V}{6 m^{*} n}$, which coincides with Equation (19) but is still larger than the value given by Equation (18). For the case of a small effective viscosity/inertia, $\beta \gg 1$, we find $\delta F_{L}(t \rightarrow \infty) \simeq-\frac{\lambda v^{4} V}{24 m^{*} n}$, which is much higher than the free energy given by both stationary solutions expressed in Equations (18) and (19). Thus, one may expect that Equation (33) either describes a metastable state or a state that slowly varies on a time scale $t_{k} \gg t_{\gamma} \sim 1 / \gamma_{m}$, reaching, for $t \gg t_{k}$, the stationary state with the free energy given by Equation (18). To show the latter possibility, consider the case $\beta \gg 1$ and assume $k$ in Equation (29) to be a slow function of time, i.e., $k=k(t)$, for a typical time scale $t_{k} \gg t_{\gamma}$. One can see that, for $R_{\text {seed }} \ll t_{k}\left|u_{T}\right|$, the quantity $k(t)$ satisfies $\left(d^{2} k / d t^{2}\right)=-k^{2} \tilde{\eta}(d k / d t)$ with the solution

$$
k(t)=k_{00}\left[1+\tilde{\eta} \lambda v^{2} t /(3 c)\right]^{-1 / 2}
$$

such as $k(t \rightarrow \infty) \rightarrow 0$, and the free energy for $t \rightarrow \infty$ indeed reaches the limit expressed in Equation (18) provided we set $\sin \chi \simeq \frac{\sqrt{3}}{2}-\frac{\sqrt{3} m^{*} \tilde{\epsilon}}{8}$. From Equation (34), we easily find that the typical time scale is $t_{k} \sim \beta t_{0}$, and we confirm that indeed $t_{k} \gg t_{\gamma}$. For $R_{\text {seed }} \gtrsim t_{k}\left|u_{T}\right| \sim l \sqrt{\beta}$, the solution expressed in Equation (29) with Equation (34) does not hold and should be modified.

For $\beta \ll 1, k_{0}=0$, Equation (34) with a slowly varying $k(t)$ does not hold. At realistic conditions, convection and sticking processes (at sizes $\sim l$ ) may be allowed, which destroy periodicity, and owing to these processes the system may finally reach the ground state with the free energy given by Equation (18). Thus, one possibility is that, for the typical time $t \sim t_{\gamma} \sim t_{0}$, the quasiperiodic solution expressed in Equation (33) is formed with a typical $k \simeq k_{m}$, corresponding to a metastable state with the free energy given by Equation (19). Such a distribution is formed most rapidly. Another possibility is that, for the typical time scale $t_{\text {unif }}>t_{\gamma}$ in a system of a large size, an approximately uniform solution, expressed in Equation (35), is developed. In the latter case, to proceed, consider the case $k \sim 1 / R \ll k_{m}$, where $R$ is the typical size of the system $\left(R=R_{\mathrm{f} .0}\right.$. for the fireball formed in heavy-ion collisions). The spatially uniform solution of

$$
\Delta_{\tilde{\zeta}} \psi+2 \psi\left(1-\psi^{2}\right)+\tilde{\epsilon}=\partial_{\tau} \psi,
$$

which follows from Equation (8) in this case (as well as for seeds of a size $R_{\text {seed }} \ll R$ at $\beta \ll 1$, as we have argued above), is given by

$$
\psi(t)= \pm 1 / \sqrt{1+e^{-\tau}\left(1-\psi_{0}^{2}\right) / \psi_{0}^{2}},
$$

where we, for simplicity, have $\tilde{\epsilon} \rightarrow 0$. The typical time needed for the initial amplitude $\psi_{0} \ll 1$ to grow to $\psi(t \rightarrow \infty) \simeq \pm 1$ is $t_{\text {unif }} \sim t_{0} \ln \left(1 / \psi_{0}^{2}\right) \gg t_{\gamma}$.

Thus, we found some novel solutions describing the evolution of fluctuations in the region of instability in addition to the uniform solution expressed in Equation (35). For $k=$ const $\neq 0$, we found periodic solutions given by Equations (29) and (31). For $k=k_{0} \neq 0$, the solution yields the minimum of the free energy for $t \rightarrow \infty$. For $k_{0}=0, \beta \gg 1$, we found quasiperiodic solutions expressed in Equations (29) and (31) with $k=k(t)$ from Equation (34), yielding the minimum of the free energy for $t \rightarrow \infty$.

\section{Conclusions}

According to our findings, signatures of QCD spinodal instabilities might in principle be observed in experiments with heavy ions in a collision energy interval that corresponds to the first-order phase 
transition region of the QCD phase diagram. If the typical times of the growth of a fluctuation in the unstable region $t_{\gamma}$ and of that of the fireball expansion $t_{\text {f.o. }}$ satisfy the condition $t_{\gamma} \lesssim t_{\text {f.o., }}$ one of the possible experimental signatures of the spinodal region would be a manifestation of a spatially quasiperiodic structure with a typical period $r \simeq 2 \pi / k_{m}$. If the parameter characterizing effective viscosity/inertia $\beta$ were $\gg 1$, cf. Equation (12), then for $t_{\gamma} \ll t_{\text {f.o. }}$ one of the possible experimental signatures of the spinodal region would be a manifestation of spatially quasiperiodic fluctuations with a typical size $r \sim 2 \pi / k\left(t_{\text {f.o. }}\right) \gg 2 \pi / k_{m}$. However, rough estimates made for the quark-hadron and nuclear gas-liquid first-order phase transitions in heavy-ion collisions [54-56] indicate that $\beta \ll 1$. Future experimental programs at NICA and FAIR will scan the collision energy interval, in which various manifestations of the first-order quark-hadron phase transition are expected, including possible signatures of quasiperiodic structures. It would also be interesting to search for the consequences of the possible formation of quasiperiodic structures during the quark-hadron phase transition in the early universe.

Concluding, we note that viscosity and thermal conductivity are the driving forces of the first-order liquid-gas and quark-hadron phase transitions to the state with $k_{0}=0$, and the spinodal instability occurs for $T$ below the ITS line. The manifestation of a spatially quasiperiodic structure with a typical period of $2 \pi / k_{m}$, cf. Equation (29), in the rapidity spectra of heavy-ion collisions in a collision energy interval could be interpreted as a signature of the occurrence of the spinodal instability at the first-order phase transition. For the second-order phase transition to the state with $k_{0} \neq 0$, as for the case of the pion condensation in dense nuclear matter, the periodic solution expressed in Equation (29) holds for $k=k_{0} \neq 0$, where $k_{0}$ does not depend on time.

Funding: This research received no external funding.

Acknowledgments: I thank E. E. Kolomeitsev for the discussions.

Conflicts of Interest: The author declares no conflict of interest.

\section{References}

1. Aghanim, N.; Akrami, Y.; Ashdown, M.; Aumont, J.; Baccigalupi, C.; Ballardini, M.; Banday, A.J.; Barreiro, R.B.; Bartolo, N.; Basak, S.; et al. Planck Collaboration. arXiv 2018, arXiv:1807.06209.

2. Linde, A.D. Phase Transitions in Gauge Theories and Cosmology. Rept. Prog. Phys. 1979, 42, 389. [CrossRef]

3. Witten, E. Cosmic separation of phases. Phys. Rev. D 1984, 30, 272. [CrossRef]

4. Rafelski, J. Connecting QGP-Heavy Ion Physics to the Early Universe. Nucl. Phys. Proc. Suppl. 2013, $243,155$. [CrossRef]

5. Busza, W.; Rajagopal, K.; van der Schee, W. Heavy Ion Collisions: The Big Picture, and the Big Questions. Ann. Rev. Nucl. Part. Sci. 2018, 68, 339. [CrossRef]

6. Jacak, B.V.; Muller, B. The exploration of hot nuclear matter. Science. 2012, 337, 310. [CrossRef]

7. Fodor, Z. Selected results in lattice quantum chromodynamics. PTEP 2012, 2012, 01A108. [CrossRef]

8. Bazavov, A.; Ding, H.T.; Hegde, P.; Kaczmarek, O.; Karsch, F.; Karthik, N.; Laermann, E.; Lahiri, A.; Larsen, R.; Li, S.T.; et al. Chiral crossover in QCD at zero and non-zero chemical potentials. Phys. Lett. B 2019, 795, 15-21. [CrossRef]

9. Kapusta, J.; Gale, C. Finite-Temperature Field Theory Principles and Applications; Cambridge Univ. Press: Cambridge, UK, 2006.

10. Peebles, P.J.E. Principles of Physical Cosmology; Princeton Univ. Press: Princeton, NJ, USA, 1993.

11. Shapiro, S.; Teukolsky, S.A. Black Holes, White Dwarfs and Neutron Stars: The Physics of Compact Objects; Wiley: New York, NY, USA, 1983.

12. Migdal, A.B.; Saperstein, E.E.; Troitsky, M.A.; Voskresensky, D.N. Pion degrees of freedom in nuclear matter. Phys. Rept. 1990, 192, 179. [CrossRef]

13. Haubold, H.; Kampfer, B.; Senatorov, A.; Voskresensky, D. A Tentative approach to the second neutrino burst in SN1987A. Astron. Astrophys. 1988, 191, L22.

14. Galeotti, P.; Pizzella, G. New analysis for the correlation between gravitational wave and neutrino detectors during SN1987A. Eur. Phys. J. C 2016, 76, 426. [CrossRef] 
15. Fischer, T.; Bastian, N.U.; Wu, M.R.; Baklanov, P.; Sorokina, E.; Blinnikov, S.; Typel, S.; Klähn, T.; Blaschke, D.B. Quark deconfinement as a supernova explosion engine for massive blue supergiant stars. Nat. Astron. 2018, 2, 980-986. [CrossRef]

16. Prakash, M.; Bombaci, I.; Prakash, M.; Ellis, P.; Lattimer, J.; Knorren, R. Composition and structure of protoneutron stars. Phys. Rept. 1997, 280, 1. [CrossRef]

17. LIGO Scientific and Virgo and 1M2H and Dark Energy Camera GW-E and DES and DLT40 and Las Cumbres Observatory and VINROUGE and MASTER Collaborations. A gravitational-wave standard siren measurement of the Hubble constant. Nature 2017, 551, 85. [CrossRef]

18. Demorest, P.; Pennucci, T.; Ransom, S.; Roberts, M.; Hessels, J. Shapiro delay measurement of a two solar mass neutron star. Nature 2010, 467, 1081. [CrossRef]

19. Fonseca, E.; Pennucci, T.T.; Ellis, J.A.; Stairs, I.H.; Nice, D.J.; Ransom, S.M.; Demorest, P.B.; Arzoumanian, Z.; Crowter, K.; Dolch, T.; et al. The NANOGrav nine-year data set: Mass and geometric measurements of binary millisecond pulsars. Astrophys. J. 2016, 832, 167. [CrossRef]

20. Antoniadis, J.; Freire, P.C.C.; Wex, N.; Tauris, T.M.; Lynch, R.S.; van Kerkwijk, M.H.; Kramer, M.; Bassa, C. A massive pulsar in a compact relativistic binary. Science 2013, 340, 1233232. [CrossRef]

21. Cromartie, H.T.; Fonseca, E.; Ransom, S.M.; Demorest, P.B.; Arzoumanian, Z.; Blumer, H.; Brook, P.R.; DeCesar, M.E.; Dolch, T.; Ellis, J.A.; et al. Relativistic Shapiro delay measurements of an extremely massive millisecond pulsar. Nat. Astron. 2019, 4, 72. [CrossRef]

22. Bauswein, A.; Bastian, N.; Blaschke, D.; Chatziioannou, K.; Clark, J.; Fischer, T.; Oertel, M. Identifying a first-order phase transition in neutron star mergers through gravitational waves. Phys. Rev. Lett. 2019, 122, 061102. [CrossRef]

23. Shuryak, E. What RHIC experiments and theory tell us about properties of quark-gluon plasma? Nucl. Phys. A 2005, 750, 64. [CrossRef]

24. Teaney, D.; Lauret, J.; Shuryak, E. A Hydrodynamic description of heavy ion collisions at the SPS and RHIC. arXiv 2001, arXiv:nucl-th/0110037.

25. Romatschke, P.; Romatschke, U. Viscosity Information from Relativistic Nuclear Collisions: How Perfect is the Fluid Observed at RHIC? Phys. Rev. Lett. 2007, 99, 172301. [CrossRef] [PubMed]

26. Shuryak, E. Physics of Strongly coupled Quark-Gluon Plasma. Prog. Part. Nucl. Phys. 2009, 62, 48. [CrossRef]

27. Röpke, G.; Münchow, L.; Schulz, H. Particle clustering and Mott transitions in nuclear matter at finite temperature. Nucl. Phys. A 1982, 379, 536. [CrossRef]

28. Schulz, H.; Voskresensky, D.N.; Bondorf, J. Dynamical aspects of the liquid-vapor phase transition in nuclear systems. Phys. Lett. B 1983, 133, 141. [CrossRef]

29. Chomaz, P.; Colonna, M.; Randrup, J. Nuclear spinodal fragmentation. Phys. Rept. 2004, 389, 263. [CrossRef]

30. Margueron, J.; Chomaz, P. A Unique spinodal region in asymmetric nuclear matter. Phys. Rev. C 2003, 67, 041602. [CrossRef]

31. Maslov, K.; Voskresensky, D. RMF models with $\sigma$-scaled hadron masses and couplings for the description of heavy-ion collisions below 2 A GeV. Eur. Phys. J. A 2019, 55, 100. [CrossRef]

32. avenhall, D.G.; Pethick, C.J.; Wilson, J.R. Structure of Matter Below Nuclear Saturation Density. Phys. Rev. Lett. 1983, 50, 2066. [CrossRef]

33. Maruyama, T.; Tatsumi, T.; Voskresensky, D.N.; Tanigawa, T.; Chiba, S. Nuclear pasta structures and the charge screening effect. Phys. Rev. C 2005, 72, 015802. [CrossRef]

34. Migdal, A.B. Pion fields in nuclear matter. Rev. Mod. Phys. 1978, 50, 107. [CrossRef]

35. Glendenning, N. Phase transitions and crystalline structures in neutron star cores. Phys. Rept. 2001, $342,393$. [CrossRef]

36. Maruyama, T.; Tatsumi, T.; Voskresensky, D.; Tanigawa, T.; Endo, T.; Chiba, S. Finite size effects on kaonic pasta structures. Phys. Rev. C 2006, 73, 035802. [CrossRef]

37. Voskresensky, D.N. On the possibility of the condensation of the charged rho meson field in dense isospin asymmetric baryon matter. Phys. Lett. B 1997, 392, 262. [CrossRef]

38. Glendenning, N. First order phase transitions with more than one conserved charge: Consequences for neutron stars. Phys. Rev. D 1992, 46, 1274. [CrossRef] [PubMed]

39. Heiselberg, H.; Pethick, C.; Staubo, E. Quark matter droplets in neutron stars. Phys. Rev. Lett. 1993, 70, 1355. [CrossRef]

40. Voskresensky, D.; Yasuhira, M.; Tatsumi, T. Charge screening at first order phase transitions and hadron quark mixed phase. Nucl. Phys. A 2003, 723, 291. [CrossRef] 
41. Maslov, K.; Yasutake, N.; Ayriyan, A.; Blaschke, D.; Grigorian, H.; Maruyama, T.; Tatsumi, T.; Voskresensky, D. Hybrid equation of state with pasta phases and third family of compact stars. Phys. Rev. C 2019, 100, 025802. [CrossRef]

42. Sedrakian, A.; Clark, J.W. Superfluidity in nuclear systems and neutron stars. Eur. Phys. J. A 2019, 55, 167. [CrossRef]

43. Kolomeitsev, E.E.; Voskresensky, D.N. Superfluid nucleon matter in and out of equilibrium and weak interactions. Phys. Atom. Nucl. 2011, 74, 1316. [CrossRef]

44. Voskresensky, D.N. Vector-boson condensates, spin-triplet superfluidity of paired neutral and charged fermions, and $3 P_{2}$ pairing of nucleons. arXiv 2019, arXiv:1911.07502.

45. Alford, M.G.; Schmitt, A.; Rajagopal, K.; Schäfer, T. Color superconductivity in dense quark matter. Rev. Mod. Phys. 2008, 80, 1455. [CrossRef]

46. Voskresensky, D.N. The phase transition to an inhomogeneous condensate state. Phys. Scr. 1984, $29,259$. [CrossRef]

47. Voskresensky, D.N. Quasiclassical description of condensed systems by a complex order parameter. Phys. Scr. 1993, 47, 333. [CrossRef]

48. Buballa, M.; Carignano, S. Inhomogeneous chiral condensates. Prog. Part. Nucl. Phys. 2015, 81, 39. [CrossRef]

49. Kolomeitsev, E.E.; Voskresensky, D.N. Negative kaons in dense baryonic matter. Phys. Rev. C 2003, 68, 015803. [CrossRef]

50. Tilley, D.R.; Tilley, J. Superfluidity and Superconductivity; Van Nostrand Reinhold Comp.: New York, NY, USA, 1974.

51. Ratra, B.; Peebles, P.J.E. Cosmological Consequences of a Rolling Homogeneous Scalar Field. Phys. Rev. D 1988, 37, 3406. [CrossRef]

52. Agarwal, S.; Corasaniti, P.S.; Das, S.; Rasera, Y. Small scale clustering of late forming dark matter. Phys. Rev. D 2015, 92, 063502. [CrossRef]

53. Chowdhury, T.A.; Nemevsek, M.; Senjanovic, G.; Zhang, Y. Dark Matter as the Trigger of Strong Electroweak Phase Transition. J. Cosmol. Astropart. Phys. 2012, 1202, 029. [CrossRef]

54. Skokov, V.V.; Voskresensky, D.N. Hydrodynamical description of a hadron-quark first-order phase transition. JETP Lett. 2009, 90, 223. [CrossRef]

55. Skokov, V.V.; Voskresensky, D.N. Hydrodynamical description of first-order phase transitions: Analytical treatment and numerical modeling. Nucl. Phys. A 2009, 828, 401. [CrossRef]

56. Skokov, V.V.; Voskresensky, D.N. Thermal conductivity in dynamics of first-order phase transition. Nucl. Phys. A 2010, 847, 253. [CrossRef]

57. Steinheimer, J.; Randrup, J. Spinodal density enhancements in simulations of relativistic nuclear collisions. Phys. Rev. C 2013, 87, 054903. [CrossRef]

58. Steinheimer, J.; Randrup, J. Spinodal amplification and baryon number fluctuations in nuclear collisions at NICA. Eur. Phys. J. A 2016, 52, 239. [CrossRef]

59. Ruggeri, F.; Friedman, W. Nucleation rate of hadron bubbles in baryon - free quark - gluon plasma. Phys. Rev. D 1996, 53, 6543. [CrossRef]

60. Voskresensky, D.N. Hydrodynamics of Resonances. Nucl. Phys. A 2011, 849, 120. [CrossRef]

61. Mandelstam, L.I.; Leontovich, M.A. To the theory of sound absorption in liquids. Z. Eksp. Teor. Fiz. 1937, 7, 438.

62. Landau, L.D.; Lifshitz, E.M. Fluid Mechanics; Pergamon Press: Oxford, UK, 1987.

63. Kolomeitsev, E.E.; Voskresensky, D.N. Viscosity of neutron star matter and $r$-modes in rotating pulsars. Phys. Rev. C 2015, 91, 025805. [CrossRef]

64. Langer, J.S. Statistical theory of the decay of metastable states. Ann. Phys. 1969, 54, 258. [CrossRef]

65. Langer, J.S.; Turski, L.A. Hydrodynamic model of the condensation of a vapor near its critical point. Phys. Rev. A 1973, 8, 3230. [CrossRef]

66. Lifshiz, E.M.; Pitaevskii, L.P. Physical Kinetics; Pergamon Press: Oxford, UK, 1981.

67. Pethick, C.J.; Ravenhall, D.G. Instabilities in hot nuclear matter and the fragmentation process. Nucl. Phys. A 1987, 471, 19. [CrossRef]

(c) 2020 by the author. Licensee MDPI, Basel, Switzerland. This article is an open access article distributed under the terms and conditions of the Creative Commons Attribution (CC BY) license (http:/ / creativecommons.org/licenses/by/4.0/). 